\title{
Asociaciones excitatorias entre el contexto y la consecuencia en la reinstalación de respuestas extinguidas en el aprendizaje causal humano
}

\section{Excitatory context-consequence associations in the reinstatement of extinguished responses in human causal learning}

\author{
Ximena A. Norambuena \\ Gonzalo R. Quintana \\ Fernando P. Ponce \\ Edgar H. Vogel \\ Universidad de Talca, Chile. \\ (Rec: 31 octubre 2009 / Acep: 10 diciembre 2009)
}

\begin{abstract}
Resumen
En dos experimentos, estudiantes universitarios aprendieron una relación predictiva entre un evento y una consecuencia, la que posteriormente fue extinguida presentando el evento sin la consecuencia. En el Experimento 1, se presentó la consecuencia por sí sola después de la extinción, ocasionando la reaparición de la relación predictiva aprendida originalmente, asemejándose al fenómeno del condicionamiento Pavloviano conocido como "reinstalación”. Este experimento demostró además, que no es necesario apelar a asociaciones inhibitorias para explicar la reinstalación, sino que solamente a asociaciones excitatorias entre el contexto y la consecuencia. El Experimento 2 confirmó la generalidad de estos hallazgos utilizando otro procedimiento de aprendizaje causal.

Se discuten estos hallazgos en términos de las diferencias entre el aprendizaje causal y el condicionamiento Pavloviano y de la posible existencia de dos mecanismos alternativos de extinción: desaprendizaje para extinguir asociaciones no consolidadas e inhibición para las consolidadas.
\end{abstract}

Palabras clave: Aprendizaje causal, Condicionamiento Pavloviano, Extinción, Reinstalación.

\begin{abstract}
In two experiments, undergraduates learned a predictive relationship between an event and a consequence, which was subsequently extinguished by presenting the event without the consequence. In Experiment 1 , participants were exposed to the consequence by itself after extinction, occasioning the reappearance of the originally learned predictive relationship, resembling a phenomenon known as Reinstatement in the field of Pavlovian conditioning. This experiment further demonstrated that reinstatement can be explained without appealing to inhibitory associations, but only by mean of excitatory associations between the context and the consequence. Experiment 2 confirmed the generality of these findings using a different procedure of causal learning.

The findings are discussed in terms of differences between Pavlovian conditioning and causal learning and of the possible existence of two mechanisms of extinction: unlearning to extinguish non consolidated associations and inhibition for the consolidated associations.
\end{abstract}

Keywords: Causal learning, Pavlovian conditioning, Extinction, Reinstatement

\footnotetext{
Correspondencia: Edgar H. Vogel. Escuela de Psicología, Universidad de Talca, Casilla 747, Talca, Chile. E-mail: evogel@utalca.cl El presente estudio está enmarcado en el Programa de Investigación "Calidad de Vida y Ambientes Saludables" de la Facultad de Psicología financiado por la Dirección de Programas de Investigación de la Universidad de Talca, Chile.
} 


\section{Introducción}

Una de las habiliddes más básicas de los seres vivos es su capacidad para establecer relaciones de predictivas y de causalidad, donde, por ejemplo, se aprende que determinados olores predicen el peligro, que una cierta configuración del cielo predice lluvia, que la actitud corporal de una persona predice su conducta, que el sabor de una sustancia predice una enfermedad estomacal o que la marca de un producto predice su calidad.

La psicología básica ha estudiado este fenómeno a través de experimentos que tienen la siguiente estructura general: se presenta a los participantes información acerca de situaciones en que una serie de eventos (como comer determinados alimentos) son seguidos o no por una consecuencia (como desarrollar una alergia), para luego preguntarles hasta qué nivel estiman que los eventos predicen tal consecuencia (cuáles alimentos producen alergia y cuáles no). Producto de este procedimiento, los juicios causales de los participantes se ajustan progresivamente a las probabilidades reales programadas para la ocurrencia de la consecuencia dados los eventos antecedentes.

Los investigadores han elaborado al menos dos tipos de teorías para explicar este "aprendizaje causal o predictivo". El primero de ellos, conocido como enfoque "cognitivo" (o normativo) propone que tanto los seres humanos como el resto de los animales realizan cálculos acerca de las relaciones entre las causas y los efectos, para luego emitir sus juicios utilizando este cálculo como parte de un esquema causal, en el que se compara la probabilidad de obtener un resultado dada una determinada clave con la probabilidad dada su ausencia. Así, este mecanismo dependería de ciertas capacidades cognitivas relativamente complejas para generar representaciones mentales de la ocurrencia de eventos y sus respectivas probabilidades (Cheng y Holyoak, 1995).

El segundo enfoque, conocido como enfoque "asociativo", plantea que el aprendizaje causal es un caso especial de condicionamiento clásico, en el cual se presentan pareados dos estímulos, uno biológicamente significativo, llamado estímulo incondicionado (EI) y uno neutro, llamado estímulo condicionado (EC). Producto de este pareamiento, el EC adquiere propiedades excitatorias que le permiten generar una respuesta condicionada (RC) que anticipa (o predice) la llegada del EI. Según la visión asociativa, el establecimiento de relaciones causales podría describirse simplemente como el desarrollo de conexiones asociativas ente los eventos predictores (ECs) y la consecuencia (EI).

La idea de que el aprendizaje causal es una instancia de aprendizaje asociativo tiene varias ventajas sobre la visión cognitiva, siendo la principal su mayor parsimonia (no apela a mecanismos cognitivos complejos, sino solamente a la asociación) y su evidente mayor plausibilidad biológica, a través de fenómenos asociativos neuronales tales como la potenciación de largo plazo (Kelso y Brown,
1986). Además, el enfoque asociativo ha recibido bastante apoyo experimental, donde se ha visto que el aprendizaje de relaciones causales progresa gradualmente describiendo curvas similares a las obtenidas con los procedimientos de adquisición de la respuesta condicionada en experimentos de condicionamiento clásico (Allan, 1993; Dickinson, Shanks y Evenden, 1984). También se ha demostrado que la extinción sigue un curso similar en tareas de juicios causales que en el condicionamiento. Específicamente, se ha observado que una vez que se ha aprendido una relación predictiva entre dos eventos, esta puede ser revertida gradualmente por medio de la presentación de la clave predictiva en ausencia de la consecuencia (Vila, 2000).

Dentro de la perspectiva asociativa, existen aún diferentes controversias que no han sido completamente abordadas en tareas de aprendizaje causal humano. Una de ellas se refiere a la naturaleza de lo que realmente ocurre durante la extinción. Por una parte, se ha propuesto que la extinción es un proceso en el cual las conexiones establecidas entre la causa y la consecuencia (llamadas conexiones excitatorias) se debilitan o desaparecen, en lo que podría llamarse "desaprendizaje" (Rescorla y Wagner, 1972). Otros enfoques proponen que las asociaciones excitatorias que se forman nunca se borran, y que lo que realmente ocurre durante la extinción es la construcción de nuevas asociaciones de signo contrario o inhibitorias, que se oponen a las excitatorias produciendo un resultado conductual nulo (Pearce y Hall, 1980; Wagner, 1981).

Las revisiones recientes en el tema de la extinción sugieren que tanto la hipótesis del desaprendizaje como la de las asociaciones inhibitorias poseen evidencia a favor y en contra (Bouton, 2004; Myers y Davis, 2007). El apoyo para la hipótesis de la inhibición proviene de hallazgos que muestran que una respuesta condicionada extinguida puede recuperarse por medio de diferentes procedimientos, que muestran que la asociación entre el EC y el EI no ha sido eliminada. Las formas de recuperación más comunes son: a) cuando los participantes son expuestos a varias presentaciones no señaladas del EI por sí sólo después de la extinción (reinstalación), b) cuando la extinción es llevada a cabo en un contexto distinto al de la adquisición y luego se realiza una prueba en el contexto original (renovación) y c) por el mero paso del tiempo (recuperación espontánea). Para explicar cabalmente estos efectos de recuperación post-extinción, se ha postulado además que las asociaciones inhibitorias que se forman durante la extinción son mucho más frágiles que las asociaciones excitatorias que se forman durante la adquisición. Esta fragilidad las haría más susceptibles al paso del tiempo o al cambio de contexto.

Pese a la innegable existencia de estos fenómenos de recuperación en el condicionamiento clásico, hay un cuerpo creciente de evidencia que señala que éstos no necesariamente ocurren en todas las circunstancias. Por ejemplo Myers, Ressler y Davis (2006), demostraron que 
EN EL APRENDIZAJE CAUSAL HUMANO

Tabla 1. Diseño del Experimento 1

\begin{tabular}{cccccc}
\hline Adquisición & $\begin{array}{c}\text { Test de } \\
\text { adquisición }\end{array}$ & Extinción & $\begin{array}{c}\text { Test de } \\
\text { extinción }\end{array}$ & Reinstalación & $\begin{array}{c}\text { Test de } \\
\text { reinstalación }\end{array}$ \\
\hline$(\mathrm{A}+)$ & $(\mathrm{A})$ & $(\mathrm{A}-)$ & $(\mathrm{A})$ & $(+)$ & $(\mathrm{A})$ \\
{$[\mathrm{B}+]$} & {$[\mathrm{B}]$} & {$[\mathrm{B}-]$} & {$[\mathrm{B}]$} & & {$[\mathrm{A}]$} \\
$(\mathrm{C}-)$ & & & & & $(\mathrm{B})$ \\
{$[\mathrm{D}-]$} & & & & {$[\mathrm{B}]$} \\
& & & & & $(\mathrm{)})$ \\
& & & & {$[\mathrm{]}$}
\end{tabular}

Nota. Se utilizó una tarea de juicios en la que los participantes debían juzgar la probabilidad de que un determinado alimento consumido en cierto Restaurant produjera problemas gastrointestinales. Las letras "A", "B", "C" y "D" indican diferentes alimentos: "Pescado", "Carne", "Helado" y "Champiñones"; mientras que los paréntesis “( )" y "[ ]" señalan dos tipos de Restaurant: "Gourmet” y "Sabores". Los símbolos “+” y "-" muestran la presencia y ausencia de problemas gastrointestinales.

la extinción del condicionamiento de miedo en ratas está mediada por la construcción de asociaciones inhibitorias, si el intervalo de tiempo entre adquisición y extinción es largo, pero que ésta resulta en desaprendizaje si el intervalo es corto. Asimismo, se ha demostrado que los correlatos neuronales de ciertos procedimientos de extinción (generalmente inmediatos), desembocan en el desarrollo de depresión de largo plazo, es decir, construcción de inhibición, mientras que en otros casos (generalmente con extinción demorada) lo que ocurre es depontenciación neuronal, es decir un retorno a niveles neutrales de activación (Cain, Godsil, Jami, Barad, 2005; Mao, Hsiao y Gean, 2006). La conclusión más probable en la actualidad es que la extinción representa tanto desaprendizaje como aprendizaje inhibitorio y que uno y otro dependerían del intervalo entre las fases de adquisición y extinción (Barad, 2006; Myers y Davis, 2007).

Si se asume que existen múltiples procesos de extinción en el condicionamiento clásico, surge la interrogante acerca de si ocurre lo mismo en el aprendizaje causal humano. Uno podría especular que en este último tipo de procedimiento la extinción podría depender del desaprendizaje más que de la construcción de asociaciones inhibitorias. Esta suposición se relaciona con el hecho que en el condicionamiento clásico los animales son expuestos a varias sesiones de muchas presentaciones de los estímulos, lo que favorecería la consolidación de las asociaciones excitatorias, dificultando así que éstas se "borraran" por desaprendizaje y favoreciendo el desarrollo de tendencias inhibitorias, como único recurso para anular la excitación. Por el contrario, los procedimientos de aprendizaje causal se realizan en una sola sesión y con pocos ensayos, los cuales generalmente son seguidos inmediatamente por ensayos de extinción. En este último caso, es más plausible argüir que los participantes son sometidos a extinción cuando aún no se ha consolidado el aprendizaje excitatorio, y que por lo tanto la solución más eficiente es simplemente borrar lo aprendido.

Se han realizado algunos intentos por explorar estos fenómenos de recuperación de respuestas en el aprendizaje causal humano, aunque el cuerpo de conocimientos en esta área es aún incipiente. Por ejemplo, Vila y Rosas (2001) investigaron la reinstalación en una tarea donde los participantes aprendieron inicialmente que un medicamento ficticio, A, provocaba un efecto colateral en los pacientes $(\mathrm{A}+)$, mientras que otro medicamento, $\mathrm{B}$, no provocaba tales efectos (B-). En la segunda fase del experimento, A y B se presentaron por sí solos sin la consecuencia, con lo cual se produjo extinción de los juicios causales adquiridos por A. Tanto en la primera fase (adquisición) como en la segunda (extinción), lo medicamentos fueron comprados en el mismo contexto (Farmacia X). Luego de este entrenamiento, los participantes fueron divididos en tres grupos, los cuales se sometieron a distintos procedimientos: En el grupo "Igual contexto- consecuencia" se presentó la consecuencia por sí sola en la Farmacia X, en el grupo "Distinto contextoconsecuencia" se presentó la consecuencia por sí sola en una nueva Farmacia Y, mientras que el grupo "No consecuencia" sólo recibió exposición a la Farmacia X. En la fase de prueba se pidió a los participantes que evaluaran el valor causal del medicamento A en la Farmacia X, encontrándose que los participantes del grupo "Igual contexto- consecuencia" asignaron valores causales significativamente más altos que los otros grupos. Los autores concluyeron que la mera exposición de la consecuencia en el contexto X ocasionó la reinstalación de los juicios causales extinguidos.

Es importante notar que los resultados de reinstalación de Vila y Rosas (2001) no necesariamente tienen que ser interpretados como prueba de que la extinción no borró la asociación causal del medicamento A. Es posible argüir que el contexto (Farmacia X), adquirió propiedades excitatorias durante la fase de reinstalación, las cuales se sumaron con el remanente excitatorio de la clave A, elevando los valores obtenidos en el test. El experimento no consideró algunas comparaciones críticas tales como la evaluación del valor causal de la Farmacia X por sí sola, lo cual deja interrogantes sin responder.

El objetivo de la presente investigación es ampliar y complementar los resultados de Vila y Rosas (2001), con el fin de determinar si la reinstalación en el aprendizaje casual 
humano puede ser explicada por una asociación entre el contexto y la consecuencia, o bien si es necesario apelar a mecanismos más complejos, tales como la construcción de asociaciones inhibitorias.

En importante enfatizar que la comprensión del fenómeno de la extinción tiene importantes implicaciones en diversos ámbitos de la psicología aplicada, especialmente en la psicopatología de los desórdenes de ansiedad y el trastorno por estrés post traumático (Cannistraro y Rauch, 2003; Rachman, 1989). En estas patologías, los pacientes tienen dificultad para suprimir conductas de miedo posiblemente adquiridas por condicionamiento clásico y susceptibles, por lo tanto, de ser tratadas por técnicas terapéuticas similares a la extinción experimental (Azorlosa, Johnson y McConnell, 2006; Havemans, Mulkens, Nederkoorn y Jansen, 2007). Resulta relevante entonces, comprender cuáles son los verdaderos alcances de la extinción como procedimiento para suprimir conductas aprendidas.

\section{Experimento 1}

El presente experimento intenta determinar si la reinstalación de juicios causales extinguidos puede ser explicada por la formación de asociaciones excitatorias entre el contexto y la consecuencia. Para esto se utilizó el paradigma de las alergias, en el que los participantes aprenden que la ingesta de determinados alimentos ficticios, consumidos en ciertos restaurantes, producen o no problemas gastrointestinales. El entrenamiento (ver Tabla 1) consideró las tres fases típicas de los estudios de reinstalación: adquisición, extinción y reinstalación, cada una de las cuales fue seguida por un test en el cual se examinaron los juicios de causalidad que los participantes atribuyeron a cada alimento.

Como se señala en la Tabla 1, la adquisición consistió en aprender que dos alimentos (designados como A y B) causaban un problema gastrointestinal, mientras que otros dos alimentos (designados como $\mathrm{C}$ y D) no causaban dichos problemas. Los alimentos A y $\mathrm{C}$ eran consumidos en un restaurante (designado como "( )") mientras que los alimentos B y D eran consumidos en otro restaurante (designado como "[ ]"). Con este ordenamiento de eventos y consecuencias, se esperaba que los participantes aprendan que las claves A y B son predictivas de la consecuencia, que las claves $\mathrm{C}$ y $\mathrm{D}$ no son predictivas de la consecuencia y que los restaurantes o contextos son irrelevantes. Luego, en la fase de extinción, aquellas claves que durante la adquisición se asociaron con el problema gastrointestinal, pierden dicha asociación ((A-), [B-] ). Mientras que aquellas que durante la adquisición carecían de asociación ((C-) y [D-]), se omiten. Finalmente, en la fase de reinstalación no se presentan claves a asociar, sino que sólo se presenta uno de los dos contextos junto con la consecuencia.

Se obtiene evidencia de reinstalación si los juicios causales de A en su contexto original ((A)) aumentan después de la reinstalación en comparación con lo observado después de la extinción. Si esta recuperación se debe a que el contexto adquirió propiedades excitatorias, entonces se esperaría que la respuesta a la clave $\mathrm{A}$ en su contexto original sea similar a la del contexto por sí solo. Alternativamente, si los participantes no atribuyen valor causal alguno al contexto en el último test, o bien si la causalidad atribuida a la clave A en el contexto supera a la del contexto por sí solo, se podría concluir que la reinstalación implica algo más que la mera adquisición de aprendizaje contextual.

\section{Método}

Participantes. Treinta y tres estudiantes universitarios, 10 hombres y 23 mujeres, participaron en el experimento por bonificación en una cátedra.

Procedimiento. Tanto en el entrenamiento como las correspondientes fases de prueba, los estímulos fueron presentados a través de un computador, programado con el software E-Prime versión 1.1. Las instrucciones del experimento aparecieron en una "ventana de instrucciones" con letra Arial 12 color blanco, normal y alineada a la izquierda, en un fondo azul y con interlineado sencillo.

Al comenzar el experimento, en la pantalla del computador aparecía el siguiente texto de bienvenida a la tarea:

Gracias por participar en esta investigación. En este experimento estamos estudiando los mecanismos de aprendizaje de las personas. Tu participación es anónima y voluntaria y todas tus respuestas se manejarán de manera totalmente confidencial.

\section{CLIK AQUÍ PARA CONTINUAR}

Al presionar el botón con el mouse, aparecía el siguiente texto, que describe las instrucciones generales del experimento:

En esta tarea te pediremos que imagines que eres parte de los fiscalizadores del Departamento de Control Sanitario del Servicio de Salud. Últimamente, el departamento ha recibido quejas de gente que ha tenido problemas gastrointestinales después de comer en ciertos restaurantes y se teme que algunos alimentos pueden estar contaminados. Para determinar la causa del problema, se te encomienda que entrevistes a diversas personas que consumieron distintos alimentos en distintos restaurantes. Los resultados de estas entrevistas aparecerán sucesivamente en la pantalla del computador. Verás una pantalla distinta para cada entrevista. En cada pantalla, se te mostrará una foto con alguna de las comidas que alguno de los entrevistados comió ese día y el restaurante donde lo comió, y sí te informará si tuvo o no un problema gastrointestinal. Por favor, pon atención a los distintos alimentos que comen los entrevistados y recuerda que tu tarea como fiscalizador es tratar de determinar cuáles son las causas de los problemas gastrointestinales de las personas. 
Luego de que los participantes presionaran el botón indicado, se desplegaban las siguientes instrucciones, que indicaban a los participantes las formas de emitir su juicio predictivo durante los ensayos y la posterior solicitud de puntaje de probabilidad:

Después de ver los alimentos consumidos, se te pedirá que predigas si la comida le produjo o no un problema gastrointestinal al entrevistado. Simplemente, haz CLICK en "SI" si crees que la persona tuvo un problema gastrointestinal y haz CLICK en "No" si piensas que el entrevistado no tuvo un problema gastrointestinal. Después que hagas tu predicción, el computador te informará si realmente el entrevistado tuvo un efecto gastrointestinal o no. Obviamente, al comienzo tendrás que adivinar, ya que no sabes nada acerca del entrevistado, pero esperamos que paulatinamente comiences a aprender las causas de los problemas gastrointestinales. Puedes ver este experimento como un juego y tratar de obtener tantos puntos (predicciones correctas) como puedas. Verás el porcentaje de respuestas correctas en la parte de abajo de la pantalla después de cada entrevista. Posteriormente en el experimento se te pedirá que le otorgues puntajes a la probabilidad de que cada alimento cause problemas gastrointestinales en el entrevistado, basándote en la información que has visto en los exámenes.

Posteriormente, se mostraba a los participantes el texto que sigue, el que buscaba resumir las principales instrucciones entregadas:

\section{Instrucciones finales}

En resumen, tu tarea consiste en aprender cuáles son las causas de los problemas gastrointestinales en los entrevistados.

La adquisición consistió en una serie de 24 ensayos (6 por cada clave entrenada), en el que aparecía una frase: " $E n$ el Restaurant XX el entrevistado comió:" (en el lugar de XX aparecía el nombre del restaurante Gourmet o Sabores, dependiendo del tipo de ensayo), seguido por la clave (una foto con alguno de los siguientes alimentos: carne, pescado, champiñones o helado) y un texto que señalaba: ¿Crees que el entrevistado tuvo un problema gastrointestinal?". El participante tenía la opción de elegir entre las respuestas "sí" o "no", presionando con el mouse un cuadro de texto. Una vez emitida la respuesta, se presentaba el feedback consistente en la frase "problema gastrointestinal" para las claves $(\mathrm{A}+)$ y $[\mathrm{B}+], y$ "sin problema gastrointestinal" para las claves (C-) y [D-], junto con la palabra "icorrecto!" o "incorrecto", de acuerdo a la respuesta del participante.

Entre cada ensayo se mostraba el porcentaje acumulado de aciertos, dentro de un cuadro de texto color negro ubicado en la esquina inferior derecha, con letra color blanco y durante $250 \mathrm{mseg}$.
Al finalizar la adquisición, se desplegaban las instrucciones para el test de adquisición:

A continuación queremos que estimes la probabilidad que cada alimento cause un problema gastrointestinal en los entrevistados.

Luego de que el participante presionara el botón para continuar, aparecía en la pantalla el texto: "En el Restaurant $X X$ el entrevistado comió:", bajo el cual se mostraba la imagen de un alimento y la siguiente pregunta: “¿En qué grado este alimento causa un problema gastrointestinal al entrevistado. Bajo la pregunta señalada, al centro de la pantalla y ubicados de izquierda a derecha, aparecían 11 cuadros de texto color blanco y letra negra, con los números: “0”, “10", “20”, “30”, “40”, “50”, “60”, “70”, "80", “90", "100", bajo los cuales se encontraban tres cuadros adicionales ubicados a la izquierda, al centro y a la derecha, indicando respectivamente: "definitivamente no", "posiblemente" y "definitivamente sí". Por último, bajo estos cuadros aparecía la frase: "selecciona un número entre 0 y 100 y haz clic en SIGUIENTE para evaluar la próxima comida".

Una vez completado el test de adquisición, aparecía la siguiente frase que se mantenía durante 1 minuto:

Usted ha completado la primera fase del experimento. A continuación habrá un pequeño descanso para relajarte.

Una vez transcurrido el tiempo de descanso, aparecían las instrucciones previas a la etapa de extinción:

A continuación comenzará la segunda parte del experimento. Las instrucciones para la segunda parte son las mismas que para la primera. De todas formas te las volveremos a mostrar.

La extinción, así como el test de extinción, conserva las mismas características de presentación reportadas para la adquisición. Sin embargo, en este entrenamiento las respuestas correctas o incorrectas fueron modificadas según las contingencias planteadas para éste (Tabla 1).

El entrenamiento de reinstalación consistía en una serie de 6 ensayos en lo que en la pantalla aparecía en la parte superior la frase: "El entrevistado comió en el Restaurant "GOURMET" y tuvo:", (la clave contextual entrenada siempre fue la misma). Bajo dicha afirmación aparecía, luego de 2-seg. y con la misma duración, la frase: "Problema Gastrointestinal". En este entrenamiento no se les solicitaba a los participantes que acertara sobre la ocurrencia o no de la consecuencia.

Inmediatamente después de que se completara esta etapa y con las mismas instrucciones de los test anteriores, comenzaba el test de reinstalación (de igual formato que el de adquisición y extinción), en el que aparecieron las claves entrenadas (A), [B] y ( ), junto con claves no entrenadas [A], (B) y [ ]. Luego de ser completado, se desplegaba en la pantalla el siguiente texto: 
Has completado el experimento. Por favor, avisa al experimentador. Muchas gracias por tu participación

\section{Resultados y Discusión}

La Figura 1 muestra los resultados de los tres tests realizados. Se observa el establecimiento de la relación causal, tanto para la clave (A) como la [B], luego del entrenamiento de adquisición (Figura 1a), con probabilidades mayores a 90, mientras que las claves (C) y [D] no muestran este juicio de causalidad respecto a la generación de problemas gastrointestinales, indicando que los participantes aprendieron la discriminación. Esta asociación de las claves (A) $\mathrm{y}$ [B] con la consecuencia, disminuye sustancialmente tras la fase de extinción (Figura 1b), atribuyéndoles juicios de causalidad cercanos a 15 . Se observa también una importante recuperación de probabilidades luego de la fase de reinstalación, en todos los elementos que se presentan en el contexto utilizado durante la reinstalación (") ( )"; Figura 1c), lo cual sugiere que si bien se replica el fenómeno de reinstalación, éste es causado únicamente por el valor causal adquirido por parte del contexto.

Con el fin de examinar la significancia estadística de estos efectos se realizó un ANOVA de mediciones repetidas de un factor, utilizando la media de los juicios causales atribuidos a cada clave en los 3 tests, totalizando 12 niveles. Este análisis arrojó un efecto principal significativo (F (11, $352)=63.755 ; \mathrm{p}<0.001)$.

Con el fin de examinar las comparaciones de interés, se realizaron análisis post-hoc utilizando la prueba LSD para cada diferencia. Con respecto al test de adquisición (Figura 1a), las claves asociadas a malestar gastrointestinal ((A) y [B]) difieren significativamente de aquellas que no $((\mathrm{C})$ y [D], ps< 0.001) lo cual evidenciaría la capacidad por parte de los participantes para discriminar entre claves predictivas y no predictivas. En el caso del test de extinción (Figura 1b), las pruebas post-hoc indican que las claves (A) y (B), que inicialmente reportaron altos valores de juicio causal, luego de la extinción arrojan una disminución significativa en sus respectivas medias de juicio causal con respecto al valor del test de adquisición, lo que daría cuenta de la ocurrencia de extinción (ps $<0.001)$. Es importante notar que los valores de las claves A y B luego de la extinción, todavía superan las de las claves negativas $((\mathrm{C})$ y [D]; ps $<0.05)$ ), lo cual indica que si bien la extinción ocurrió, esta no fue completa.

Finalmente, en el test de reinstalación (Figura 1c) los resultados muestran que las claves presentadas en conjunto con el contexto reinstalado ((A), (B) y ( )) difieren significativamente de sus equivalentes en el contexto no reinstalado ([A], [B] y [ ]; ps $<0.001$ ) pero no difieren significativamente entre sí ( $\mathrm{ps}>0.5)$, lo cual indicaría que el contexto se lleva todo el peso de la reinstalación.

Los resultados de este experimento aportan evidencia de que el contexto presente durante la reinstalación obtiene la fuerza asociativa necesaria para generar la recuperación de la respuesta tras la extinción. Esta idea se comprobó con el actual diseño en donde dos claves predictivas ((A) y [B]) tuvieron el mismo tratamiento experimental durante la adquisición y extinción, pero se ejecutó reinstalación sólo en el contexto de una de ellas. Junto con ello, durante el test de reinstalación, se solicitó a los participantes que

Figura 1. Media de juicios causales asignados por los participantes durante el Experimento 1.

Las barras representan el error estándar de la media.

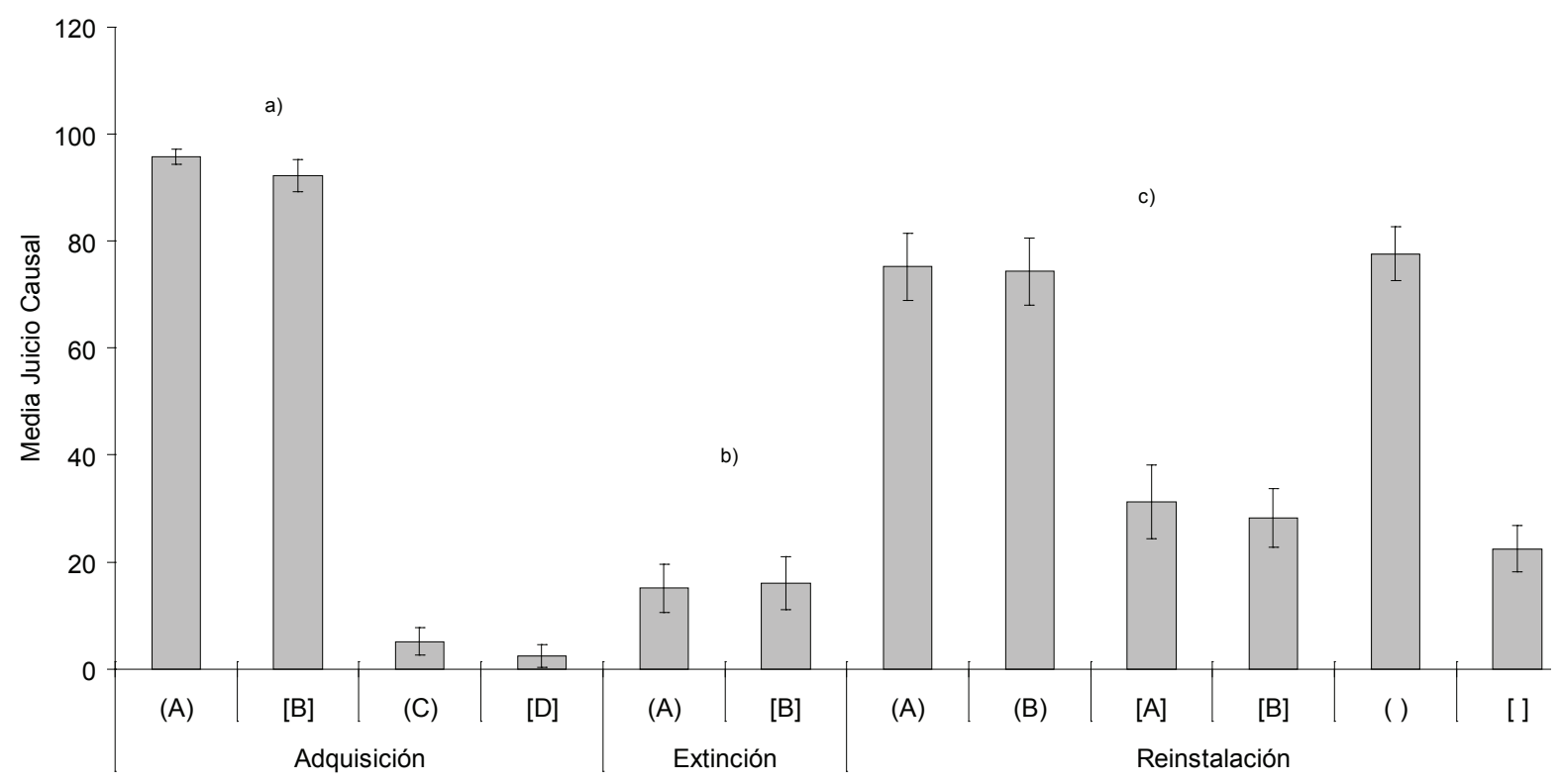


emitiesen un juicio causal frente a la presentación de cada una de las claves contextuales, en donde se pudo constatar, que los participantes emiten un juicio causal en favor de la clave contextual presente durante la reinstalación, por sobre la que no fue entrenada. El hecho fundamental de que el contexto adquiere un valor causal similar al de la clave supuestamente reinstalada (A) y al de una clave extinguida que se presenta por primera vez en ese contexto ([B]), indica que la reinstalación es un fenómeno dependiente del aprendizaje contextual excitatorio.

\section{Experimento 2}

El Experimento 1 arrojó clara evidencia en favor de que el fenómeno de reinstalación en el aprendizaje causal humano podría depender exclusivamente de la formación de asociaciones excitatorias entre el contexto y la consecuencia, al menos con los procedimientos experimentales utilizados en la presente propuesta. Esto apoyaría la noción de que en el aprendizaje causal operarían mecanismos asociativos más simples que explicarían la extinción en contraste con lo que ocurriría en los procedimientos convencionales de condicionamiento clásico con varias sesiones de aprendizaje.

Con el fin de evaluar la generalidad de estos resultados, el Experimento 2 explora el fenómeno de reinstalación en el aprendizaje causal humano utilizando un paradigma experimental distinto, en el cual los juicios causales se emiten de manera más automática y en forma no declarativa. Para ello se utilizó un procedimiento similar al de supresión condicionada, utilizado en condicionamiento Pavloviano, el que consiste en que un animal aprende a ejecutar una conducta operante (bajar una palanca), y posteriormente es sometido a un procedimiento de condicionamiento clásico, donde se parean tonos con choques eléctricos. El resultado del condicionamiento clásico implica que el animal paulatinamente desarrolla miedo al tono producto de su asociación con el choque eléctrico, lo cual ocasiona que éste, a su vez suprima la respuesta operante. Con el fin de evaluar el efecto que ejerce la presencia del estímulo condicionado (tono), los investigadores comparan la cantidad de respuestas operantes durante el tono en comparación con la cantidad de respuestas en su ausencia.

Para implementar un procedimiento de supresión condicionada en el aprendizaje causal humano se desarrolló una adaptación del paradigma planteado por Arcediano, Ortega y Matute (1996), consistente en un juego de video en el cual los participantes deben aprender a derribar naves extraterrestres que atacan el planeta presionando la barra espaciadora del teclado (el equivalente de bajar al palanca en la supresión condicionada en ratas), conducta que debe suprimirse cada vez que la nave cambia de color (el equivalente al tono de la supresión condicionada) ya que esta señal predice la venida de una consecuencia negativa si la nave es derribada (el equivalente del choque eléctrico). El aprendizaje causal, puede ser evaluado como el grado en el que los participantes aprenden progresivamente a suprimir su conducta de derribar extraterrestres en presencia de la señal en comparación con la ausencia de ésta.

El entrenamiento (Ver Tabla 2) considera cuatro fases (línea de base, adquisición, extinción y reinstalación), seguida por un test de reinstalación.

La línea de base consiste en un entrenamiento inicial en el que se busca que los participantes aprendan la conducta operante de derribar naves espaciales, presionando la barra espaciadora del computador, conducta que deberá ser ejecutada a lo largo de todo el experimento. Durante la adquisición, junto con la tarea instrumental aparecen una serie de naves de color rojo $(\mathrm{A}+)$ seguidas por la consecuencia (escudo antiláser), de tal forma que si el participante eliminase la nave que llevara el escudo recibe una gran invasión de marcianos. De esta forma los participantes progresivamente aprenderían a suprimir su conducta de derribar naves cuando la señal predictiva (A) se encuentre presente. En la extinción, las condiciones de entrenamiento fueron idénticas a las de la adquisición, sin embargo las naves rojas perdieron el valor predictivo (A-). De esta forma los participantes recuperarían la respuesta operante, previamente suprimida en la presencia de la clave. Finalmente, en la fase de reinstalación se presentó en varias ocasiones el escudo antiláser y su consecuencia sin señalización, es decir en ausencia de naves coloreadas.

Tabla 2. Diseño del Experimento 2

\begin{tabular}{cccccc}
\hline Línea de Base & Adquisición & Extinción & Reinstalación & \multicolumn{2}{c}{ Test de reinstalación } \\
\hline $100 \mathrm{LB}$ & $\mathrm{A}+$ & $\mathrm{A}-$ & + & $\mathrm{LB}$ & $\mathrm{A}$ \\
\hline
\end{tabular}

Nota. Los LB corresponden a claves neutras que no se acompañan de la consecuencia negativa y también están presentes durante las fases de Adquisición, Extinción, Reinstalación y Test. La clave A corresponde al color rojo en las naves. La consecuencia (+) corresponde a un escudo protector invisible (hasta que la nave se encuentra en la atmósfera terrestre representada por un círculo que rodea al planeta) que provoca una invasión masiva y una gran pérdida de puntaje si el marciano es eliminado mientras lo trae. 


\section{Método}

Participantes. Doce estudiantes universitarios, 3 hombres y 9 mujeres, participaron en el experimento por bonificación en una cátedra.

Procedimiento. Los materiales utilizados en el Experimento 2 fueron los mismos a los utilizados en el Experimento 1. Las instrucciones aparecían en la "ventana de instrucciones" con letra Times New Roman 12, normal, color negra y centrada, en un fondo blanco y con interlineado sencillo. Antes de comenzar el experimento, en la pantalla del computador aparecía el siguiente texto de bienvenida a la tarea:

TU PARTICIPACIÓN ES VOLUNTARIA Y ANÓNIMA

Antes que nada queremos agradecer tu participación en este experimento, ya que sin la colaboración de personas como tú no sería posible esta investigación. Lo que queremos examinar son los mecanismos básicos de aprendizaje que se dan en todas las personas. Para ello, necesitamos que si deseas participar, lo hagas con el mayor interés. No tienes que identificarte, y los datos que nos aportes se unirán al total del grupo y serán analizados estadísticamente. Es muy importante que hagas la tarea con el máximo de seriedad y atención, ya que si no lo haces, los datos del experimento se perderán. Para asegurarnos que trabajes con el máximo de motivación, la bonificación que recibirás por tu participación dependerá de la efectividad con la que realices la tarea:

Alta efectividad $=5$ décimas

Media efectividad $=2,5$ décimas

Baja efectividad $=1$ décima

SI TRAS HABER LEÍDO EL MENSAJE DESEAS CONTINUAR, PRESIONA LA BARRA ESPACIADORA.

Una vez presionada la barra espaciadora, aparecieron las instrucciones del experimento, donde se presentan las características de la tarea, basada en la idea original de Arcediano et al., (1996):

\section{INSTRUCCIONES DEL EXPERIMENTO}

$\mathrm{Tu}$ tarea es impedir el aterrizaje de los marcianos al planeta tierra. En la parte superior de la pantalla del computador aparecerá un platillo volador y en la parte inferior se verá la superficie del planeta Tierra. El platillo empezará a descender rápidamente hacia la tierra y tú tendrás que dispararle antes de que ingrese a la atmósfera de la tierra para evitar que aterrice. Los marcianos se demoran en descender aproximadamente medio segundo, y tú debes dispararles justo antes de que ingresen a la atmósfera de la tierra usando tu láser (la barra espaciadora). No te precipites, solamente asegúrate de disparar a cada marciano en el momento que te indicamos (es decir, antes que ingrese a la atmósfera). Si aciertas en tu disparo, inmediatamente aparecerá una explosión indicándote que destruiste al marciano. Posteriormente, aparecerá el planeta sin marcianos, indicando que no hubo invasión. Pero si fallas, inmediatamente aparecerá una nave sobre la superficie de la tierra y después verás un marciano parado sobre la superficie del planeta indicándote que éste logró aterrizar en la Tierra. Después de cada intento de invasión, una nueva nave comenzará a descender y tú nuevamente tendrás que intentar derribarla. Al final de cada intento, se te indicará el porcentaje total de marcianos que has derribado con tu láser hasta el momento. Tu objetivo es lograr el porcentaje más alto posible de marcianos derribados.

PRESIONA LA BARRA ESPACIADORA PARA QUE VEAS ALGUNOS EJEMPLOS DE CÓMO FUNCIONA.

Tras presionar la barra espaciadora, aparecieron en la pantalla los ejemplos sobre la tarea operante que los participantes debieron ejecutar a lo largo del experimento:

EJEMPLO 1

A continuación podrás ver un ejemplo de cuando el platillo volador es derribado. En esta oportunidad, el platillo descenderá lentamente. Esto se hizo solamente para que puedas apreciar las figuras. Sin embargo, cuando estés realizando de verdad la tarea, la velocidad aumentará considerablemente. En este ejemplo no tienes que hacer nada, sólo observa para que te familiarices con la tarea.

PRESIONA LA BARRA ESPACIADORA PARA VER ESTE EJEMPLO

Al presionar la barra, se mostró bajo el texto y con una duración de 2 segundos, un ejemplo de una nave derribada bajando a 0.5 segundos, durante cuatro momentos y sin la intervención del participante, con la correspondiente explosión y ruido. Posteriormente aparecía en la pantalla un nuevo texto, cuyo objetivo era mostrar lo que ocurre cuando el platillo no es derribado oportunamente:

\section{EJEMPLO 2}

A continuación podrás ver un ejemplo de cuando no logras derribar al marciano y la nave ingresa a la atmósfera de la tierra. En esta oportunidad, el platillo descenderá lentamente. Esto se hizo solamente para que puedas apreciar las figuras. Sin embargo, cuando estés realizando de verdad la tarea, la velocidad aumentará considerablemente. En este ejemplo no tienes que hacer nada, sólo observa para que te familiarices con la tarea.

PRESIONA LA BARRA ESPACIADORA PARA VER ESTE EJEMPLO

Al igual que en el caso anterior, después de que el participante presionara la barra espaciadora, aparecían la naves descendiendo a la misma velocidad que en el ejemplo anterior, pero esta vez el no acertarle un disparo, se mostraba la imagen de un marciano parado sobre la superficie del planeta y se escuchaba el sonido que lo acompaña. 
Posteriormente se presentaban las últimas instrucciones previas a la primera fase del experimento:

\section{INSTRUCCIONES FINALES}

Recuerda que tu tarea consiste en derribar tantos marcianos como te sea posible y así impedir que aterricen en el planeta tierra. Para derribarlos, presiona la barra espaciadora cuando el marciano esté a punto de ingresar a la atmósfera de la tierra. Recuerda que la bonificación que recibirás por tu participación dependerá de la efectividad con la que realices la tarea:

Alta efectividad $=5$ décimas

Media efectividad $=2,5$ décimas

Baja efectividad $=1$ décima

BUENA SUERTE

PRESIONA LA BARRA ESPACIADORA CUANDO ESTÉS LISTO PARA EMPEZAR

La fase de línea de base consistió en la presentación de 100 ensayos de naves incoloras no reforzadas (LB) que demoraban $500 \mathrm{mseg}$. En descender hasta el planeta, momento en el cual el participante debía derribarlo, presionando oportunamente la barra espaciadora.

En el caso de que el participante presionara la barra en el momento preciso, el marciano era eliminado, mostrándose en su lugar una explosión y el sonido que la representa. $\mathrm{Si}$ en cambio la persona no emitía la respuesta o la emitía en el momento equivocado, se mostraba la invasión del marciano a través de su llegada al planeta que se encontraba en la parte inferior de la pantalla.

Durante todos los ensayos de entrenamiento (así como en el resto del experimento), se mostraba en la pantalla un porcentaje de efectividad alcanzada por el participante, cuyo cálculo se desprende del número de naves derribadas dividido por el total de naves presentadas hasta ese ensayo.

Una vez concluidos los ensayos de línea de base, aparecía durante un lapso de 4 seg. un mensaje que daba por concluida la fase de línea de base:

LA PRIMERA PARTE HA CONCLUIDO. ESPERA UN PAR DE SEGUNDOS Y LUEGO APARECERÁN LAS INSTRUCCIONES DE LA SEGUNDA PARTE

Este es seguido por otro que presenta las instrucciones de la fase de adquisición, dando inicio a esta fase, las instrucciones advierten participante respecto al desarrollo por parte de los marcianos de un escudo antiláser y de algunos posibles indicadores de este evento:

\section{INSTRUCCIONES PARA LA SEGUNDA PARTE}

A partir de ahora, los marcianos han desarrollado un escudo anti-láser. Debes seguir disparando como hasta ahora para impedir que aterricen, pero CUIDADO, porque si disparas cuando el escudo está conectado tu disparo rebotará, la nave logrará entrar a la atmósfera y muchos marcianos aprovecharán para invadirte, y ya no podrás hacer nada para impedir esa invasión. El problema es que el escudo es INVISIBLE, y sólo se ve cuando la nave ya entró en la atmósfera terrestre, donde la nave aparecerá rodeada de un círculo negro. En conclusión, si disparas cuando el ESCUDO INIVISIBLE está conectado te ocurrirá una invasión múltiple. Primero verás la nave cubierta con su escudo negro entrando en la atmósfera y posteriormente verás a la tierra rodeada por un grupo de marcianos. Es decir, ya no verás sólo un marciano sino que varios de ellos alrededor de nuestro planeta. Además, si cometes el error de disparar cuando el platillo está con el escudo, tu porcentaje de efectividad disminuirá enormemente; mucho más que lo que disminuiría si dejaras aterrizar a un solo marciano. Afortunadamente algunos INDICADORES te ayudarán a predecir cuándo está conectado el escudo invisible. Si aprendes a distinguir los indicadores, podrás liberarte siempre del escudo. De lo contrario, cada vez que conecten el escudo te pillarán disparando y te invadirán. Recuerda, basta que realices un sólo disparo mientras el escudo está conectado para que te invadan!!! Si aprendes a detectar cuando está conectado el escudo, más te vale que dejes aterrizar a este platillo ya que si le disparas la consecuencia será mucho peor (la invasión de muchos marcianos al mismo tiempo).

PRESIONA LA BARRA ESPACIADORA PARA VER UN EJEMPLO

Una vez presionada la barra espaciadora se despliega el siguiente mensaje:

\section{EJEMPLO 3}

A continuación podrás ver un ejemplo de cuando disparas mientras la nave lleva el escudo activado ingresando a la atmósfera de la tierra. En esta oportunidad, el platillo descenderá lentamente. Esto se hizo solamente para que puedas apreciar las figuras. Sin embargo, cuando estés realizando de verdad la tarea, la velocidad aumentará considerablemente. En este ejemplo no tienes que hacer nada, sólo observa para que te familiarices con la tarea.

PRESIONA LA BARRA ESPACIADORA PARA VER ESTE EJEMPLO

Posteriormente, se mostraba en la parte inferior de la pantalla, a una velocidad de 2 segs. aproximadamente, el descenso de una nave, que finalmente fue derribada teniendo el escudo antiláser. Esta vez, se producía una invasión masiva, acompañada por un ruido característico. Posteriormente, se mostraba el siguiente texto, que buscaba sintetizar las nuevas instrucciones entregadas:

\section{INSTRUCCIONES FINALES}

Recuerda que tu objetivo es derribar la mayor cantidad posible de marcianos y así lograr un mayor porcentaje de efectividad. Pero no te olvides que TIENES QUE DEJAR DE DISPARAR CUANDO EL PLATILLO TENGA CONECTADO SU ESCUDO, ya que si le disparas vas a 
recibir la invasión de muchos marcianos y tu efectividad va a bajar enormemente. Cuando el escudo está conectado, lo mejor que puedes hacer es permitir que aterrice un sólo marciano, ya que si le disparas, las consecuencias son mucho peores. Se te darán claves que te ayuden a predecir cuando venga la nave con el escudo. Recuerda que la bonificación que recibirás por tu participación dependerá de la efectividad con la que realices la tarea:

Alta efectividad $=5$ décimas

Media efectividad $=2,5$ décimas

Baja efectividad $=1$ décima

BUENA SUERTE

PRESIONA LA BARRA ESPACIADORA CUANDO ESTÉS LISTO PARA EMPEZAR.

Cuando el participante presionaba la tecla indicada, comenzaba la etapa de adquisición, compuesta por 36 ensayos, consistentes en la presentación de 6,9 ó 12 naves incoloras (como las presentadas durante la línea de base), una serie de 3, 4 ó 5 naves rojas, en las que la última veía acompañada del escudo antiláser $(\mathrm{A}+)$. Si el participante eliminaba al último marciano de la serie de naves rojas presentada a lo largo de los ensayos, se mostraba en la pantalla una invasión masiva, representada por 13 marcianos que rodeaban el planeta, una gran pérdida de puntaje y un texto que señalaba: “iiiiERROR: INVASION MASIVA!!!! Estás en serio riesgo de perder tus décimas de bonificación". La imagen y el texto tenían una duración de $5 \mathrm{seg}$., con independencia de la conducta del sujeto, como una de las medidas introducidas para aumentar la importancia del castigo por error.

Una vez completada los ensayos de adquisición, en la pantalla aparecía el siguiente texto, que se mantenía por 3 minutos:

\section{ATENCIÓN}

A continuación tendremos un pequeño descanso. Presta atención a la pantalla del computador ya que pronto se te anunciará que tendrás que seguir con la tarea que estabas haciendo.

Luego del paso del tiempo especificado, en la pantalla se mostraba el siguiente texto, luego del cual comenzaba el entrenamiento de extinción:

\section{ATENCIÓN}

Prepárate para empezar de nuevo. Presiona la BARRA ESPACIADORA cuando estés listo para comenzar.

Tras presionar la barra espaciadora, se da inicio a la fase de extinción, consistente en 18 ensayos con las mismas características que durante la adquisición salvo que en las series de naves rojas, en que ninguna venía acompañada del escudo antiláser (A-).

Inmediatamente después de la extinción, se inició la fase de reinstalación consistente en la reaparición del escudo antiláser en 15 de un total de 135 naves sin ningún tipo de señalización y en un orden pseudoaleatorio, mientras los participantes realizaban su conducta de derribar marcianos a una tasa constante.

Tras la reinstalación, el test de reinstalación consistió en la presentación de tres ensayos de la clave predictiva (A), consistente en la presentación de cinco naves rojas cada uno, y tres ensayos de línea de base (LB) los cuales consistieron en la presentación de 5 naves incoloras cada uno. Luego de esto se agradece al participante y finaliza el experimento.

\section{Resultados y Discusión}

A diferencia del Experimento 1, donde los juicios causales se obtuvieron directamente preguntando a los participantes, en el Experimento 2 éstos tienen que inferirse indirectamente a través de su conducta. El nivel de causalidad que los participantes atribuyen a la señal está dado por el grado en el que éstos suprimen su conducta de derribar marcianos en comparación con una línea base. Considerando que la señal de interés está presente durante 5 naves consecutivas, se optó por comparar el número promedio de naves derribadas en las últimas 3 , en comparación con las últimas 3 naves que aparecieron antes del la señal (denominado línea base o LB). Así, si los participantes derriban la misma cantidad de naves con la señal que sin la señal, esto evidenciará ausencia de causalidad, mientras que si los participantes derriban menos naves en presencia de la señal que en su ausencia, esto reflejaría asociación causal. Naturalmente, la dificultad de la tarea se ajustó para que con el entrenamiento de línea base, la mayoría de los participantes derriben el $100 \%$ de las naves no señalizadas o LB. Con esto, cualquier disminución en este valor indicaría la presencia de aprendizaje causal.

Para analizar la significancia estadística de los resultados, se realizó un ANOVA de un factor, utilizando el valor promedio de naves derribadas señalizadas (en presencia de A) y no señalizadas (LB) al finalizar la adquisición, la extinción y la reinstalación. Esto arroja un factor con 6 niveles, el cual resultó estadísticamente significativo ( $F$ $(5,55)=9.495 ; p<0.001)$. La naturaleza de este efecto se puede observar en los distintos valores indicados en la Figura 2, los cuales fueron contrastados estadísticamente utilizando la prueba post hoc LSD.

La Figura 2 muestra que al finalizar la adquisición, los participantes aprendieron a suprimir su respuesta en presencia de $\mathrm{A}$, arrojando valores más bajos que los observados en la línea base $(\mathrm{p}=0.002)$. Evidencia de extinción se observa en que las respuestas de los participantes a la clave señalizada (A) al finalizar la extinción son mayores que al finalizar la adquisición $(\mathrm{p}=0.008)$ y no difieren de la línea base $(p=0.137)$. Finalmente, se aprecia cierta evidencia de reinstalación, puesto que después de esta fase los participantes responden a la clave señalizada menos que al finalizar la extinción ( $\mathrm{p}=0.026$ ). 
Es de interés notar que los resultados de la línea de base también arrojan una disminución luego de la reinstalación, lo que sugeriría que la recuperación de la supresión es un efecto generalizado que no se limita a las naves señalizadas $(\mathrm{p}=0.054)$. Asimismo, llama la atención que en el último test (Figura 2c), las diferencias entre la clave señalizada y la línea base no alcanza la significancia estadística $(p=0.139)$.

Estos hallazgos apoyan las conclusiones del Experimento 1, en tanto que si bien se obtuvo cierta evidencia de reinstalación, esta puede ser explicada por la asociación causal entre el contexto y la consecuencia. En este caso, las presentaciones no señalizadas de la nave actuarían como claves contextuales, las cuales adquieren valor causal durante la fase de reinstalación donde no existe ningún predictor más confiable de la consecuencia.

\section{Discusión General}

Tomados en conjunto, ambos experimentos proporcionan evidencia de que los juicios causales adquiridos y posteriormente extinguidos pueden ser reinstalados por la mera presentación de la consecuencia, en ausencia de las señales predictivas aprendidas originalmente. En el Experimento 1, se obtuvo reinstalación de los juicios causales con un paradigma en el cual los juicios causales fueron obtenidos de manera declarativa. En el Experimento 2, se obtuvo evidencia de reinstalación en un procedimiento en el cual el juicio de causalidad se extrajo de manera indirecta a través de la conducta de los participantes. En ambos casos, la recuperación observada para la clave extinguida es un resultado consistente con los reportados generalmente en investigaciones de aprendizaje causal humano (Vila y Rosas, 2001) y condicionamiento Pavloviano animal (Rescorla y Heth, 1975).

Además de la evidencia de reinstalación, los dos experimentos reportados aportan evidencia que sugiere que la reinstalación se debe a la formación de asociaciones excitatorias entre el contexto y la consecuencia, lo cual es perfectamente congruente con la hipótesis que señala que la extinción resulta en desaprendizaje. Específicamente, los datos de los dos experimentos muestran que los participantes probablemente desarrollan asociaciones excitatorias entre la clave y la consecuencia durante la adquisición y que las pierden durante la extinción; esto último inferido a partir del hecho que tras la reinstalación los participantes asignan valores causales similares la clave reinstalada más el contexto, que al contexto por sí solo. Estos hallazgos son congruentes con algunos hallazgos en el ámbito del condicionamiento clásico, donde la reinstalación de la respuesta de miedo ha sido demostrablemente asociada a la generación de asociaciones entre el contexto experimental y el estímulo incondicionado (Rescorla y Heth, 1975). Además, los resultados presentes contribuyen a la comprensión del fenómeno de reinstalación en aprendizaje causal humano, el cual si bien ha sido observado por otros autores, como Vila y Rosas (2001), no se habían realizado las comparaciones decisivas para determinar la naturaleza de este fenómeno.

Es importante mencionar que también existen reportes experimentales que contradicen los resultados obtenidos en

Figura 2. Media de marcianos derribados por los participantes durante el Experimento 2.

Las barras representan el error estándar de la media.

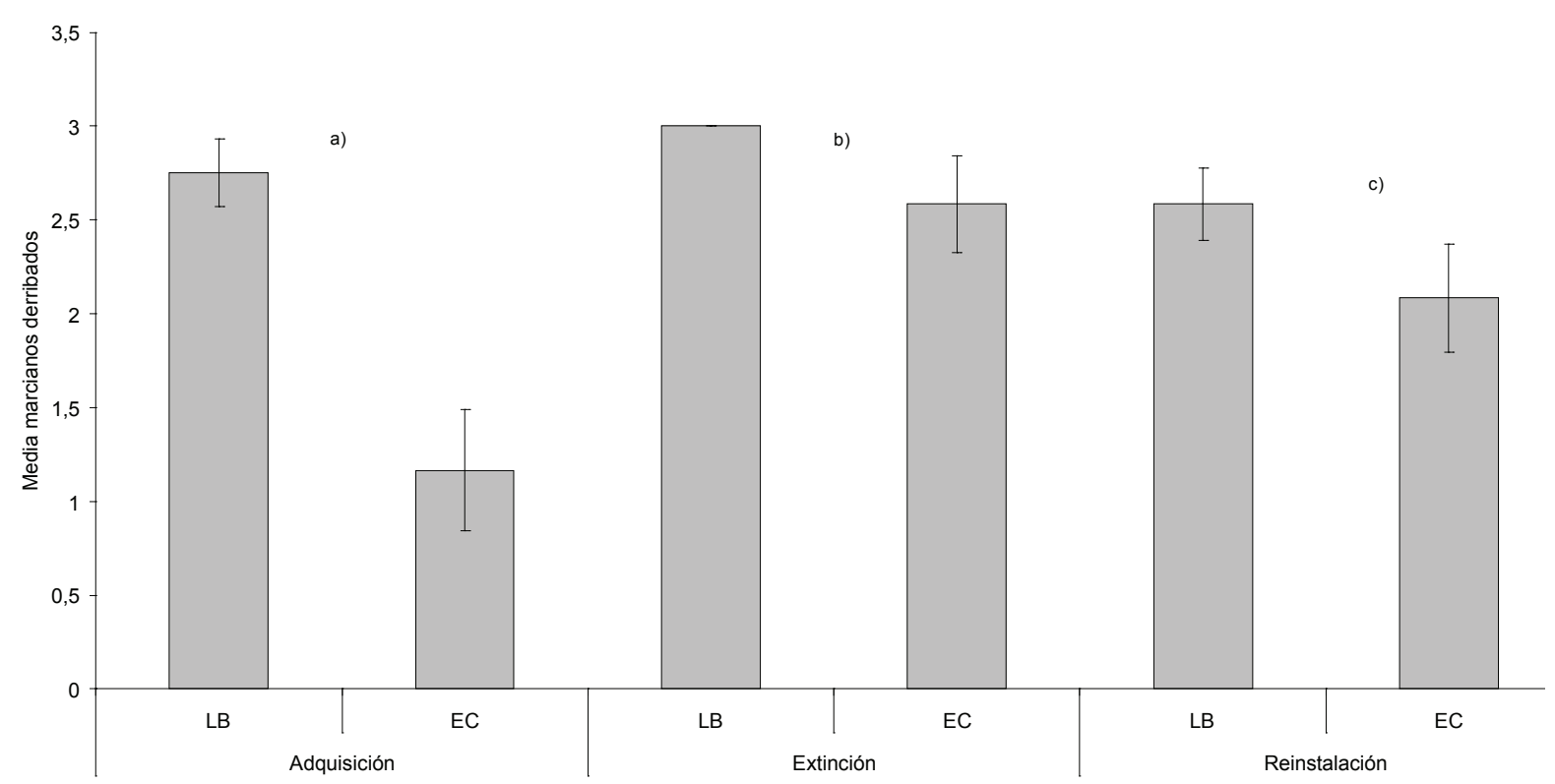


la presente investigación. Por ejemplo, García-Gutiérrez y Rosas (2003) encontraron evidencia de reinstalación en el aprendizaje de juicios causales que no podía ser explicada enteramente en términos de la adquisición de propiedades excitatorias por parte del contexto. Pese a que en este estudio no se realizó un procedimiento de extinción propiamente tal, sino de interferencia (es decir el aprendizaje de una relación predictiva entre la clave objetivo y una consecuencia incompatible con la que se aprendió originalmente), los resultados apoyan la idea de que el contexto cumpliría un rol modulador que permitiría la recuperación del material originalmente aprendido, el cual no es borrado durante la extinción sino que permanece temporalmente suprimido. Asimismo, también existe evidencia de que en el condicionamiento clásico de miedo con ratas la reinstalación se produce aún cuando el contexto por sí solo no es capaz de producir respuestas condicionadas detectables (Bouton y Bolles, 1979; Bouton y King, 1983).

Los resultados de aquellos estudios en los cuales, a diferencia del nuestro, las asociaciones excitatorias adquiridas por el contexto no son capaces de explicar totalmente la reinstalación, generan un dilema teórico que los investigadores han resuelto de diversas formas. Quizás, la propuesta más simple es aquella que sostiene que la reinstalación se debe a la combinación de dos tendencias excitatorias (el contexto y el remanente excitatorio de la clave extinguida), cada una incapaz de producir la respuesta por sí sola, pero capaces de producirla en conjunto (Hendry, 1982; Reberg, 1972). Aunque parsimoniosa, esta explicación no da cuenta de otros fenómenos de recuperación, tales como la recuperación espontánea, en el cual el mero paso del tiempo genera la recuperación de la respuesta, sin que haya alguna posibilidad de condicionamiento excitatorio por parte del contexto.

Existen alternativas teóricas que explican todos los fenómenos de recuperación (reinstalación, recuperación espontánea, y renovación) a través de mecanismos comunes que implican asumir que la extinción provoca la construcción de asociaciones inhibitorias que se oponen a las excitatorias, pero que sin embargo tienen una mayor fragilidad que éstas al ser más factibles de removerse por procedimientos tales como la reinstalación o el mero paso del tiempo. No obstante, este enfoque no da cuenta de los estudios en los cuales no se ha obtenido recuperación (Myers, Ressler y Davis, 2006) o bien se ha obtenido pero depende exclusivamente del valor excitatorio del contexto (presente estudio). Esto último hace tomar fuerza a la hipótesis de que existen múltiples mecanismos de extinción (Myers y Davis, 2007; Myers, Ressler y Davis, 2006).

La ausencia de evidencia de mecanismos inhibitorios en la presente investigación podría estar relacionada con la corta duración del intervalo transcurrido entre el entrenamiento y la extinción, favoreciendo el desaprendizaje del material no consolidado por sobre la inhibición. En este sentido, los resultados obtenidos en ambos experimentos apoyan parcialmente los hallazgos previos de Myers et al., (2006), en donde el intervalo de tiempo transcurrido entre los entrenamientos determinó el mecanismo de aprendizaje predominante durante la extinción: desaprendizaje para intervalos cortos e inhibición para intervalos largos.

Por lo anterior, seria interesante en futuras investigaciones comparar la naturaleza de lo que se aprende en la extinción de juicios causales, variando el intervalo de tiempo transcurrido entre la adquisición y la extinción. Si la hipótesis de la consolidación es cierta, uno esperaría que las respuestas reinstaladas dependan más de la reaparición de los valores excitatorios de la clave original que de la creación de asociaciones excitatorias entre el contexto y la consecuencia.

Asimismo, la posibilidad de que la extinción implique solamente desaprendizaje en los procedimientos de aprendizaje causal humano debe ser explorada a través de otros fenómenos de recuperación tales como la renovación y la recuperación espontánea, en los cuales el rol del contexto es menos claro. Estos fenómenos también deberían investigarse variando los niveles de consolidación de la información que se aprende originalmente.

Cabe una reflexión con respecto a lo que se entiende por contexto en el aprendizaje causal humano. Los contextos ficticios utilizados en la tareas de juicios causales usados en la presente investigación (restaurantes en el Experimento 1 y estimulación general en la pantalla del computador en el Experimento 2), así como también en otros contextos tales como farmacias (Vila y Rosas, 2001) y restaurantes (García-Gutiérrez y Rosas, 2003) tienen varias propiedades que los hacen más parecidos a "claves discretas" (corta duración, delimitación formal y temporal, etc.) que a las claves contextuales normalmente utilizadas en los experimentos de condicionamiento clásico (aparatos experimentales o ruidos de fondo). Este mayor parecido de las claves "contextuales" con las claves discretas en los estudios de juicios causales podría explicar, en parte, el por qué el contexto adquiere propiedades excitatorias, lo cual generalmente no ocurre en el condicionamiento clásico. Es relevante que en futuras investigaciones se contemple la utilización de claves contextuales que disten más sustancialmente de las claves predictivas que lo que se ha hecho hasta ahora en las investigaciones de aprendizaje causal. Ello contribuiría al establecimiento de las similitudes y diferencias que existen en los mecanismos que controlan el condicionamiento clásico y el aprendizaje causal.

Asimismo, la utilización de contextos más realistas permitiría establecer interesantes vínculos con estudios recientes en los cuales se ha demostrado experimentalmente la existencia de fenómenos de reinstalación de conductas fóbicas o de miedo en humanos. Por ejemplo, Norrholm, Jovanovic, Vervliet, Myers, Davis, Rothbaum y Duncan (2006) entrenaron a un grupo de personas a manifestar 
conductas de miedo ante la presencia de luces coloreadas que se parearon con una leve pero molesta inyección de aire en la garganta. Pese a que en una etapa posterior las personas fueron exitosamente "tratadas" en su miedo a las luces con un típico procedimiento de extinción, el miedo reapareció vigorosamente después de recibir unas pocas presentaciones asiladas de la inyección de aire. Por su parte, LaBar y Phelps (2005), además de demostrar la ocurrencia de reinstalación de las respuestas de miedo a estímulos asociados con ruidos molestos y choques eléctricos, presentan interesante evidencia que sugiere que los pacientes amnésicos con daño hipocampal, pese a desarrollar conductas de miedo aprendido, no presentan el fenómeno de reinstalación. Esto último apoya la idea que la reinstalación implica algún tipo de memoria acerca del contexto en el cual ocurre el evento aversivo.

La importancia de estos estudios con conductas de miedo en humanos radica en el hecho que le otorgan plausibilidad a la hipótesis que la reinstalación, como fenómeno propio de la psicología básica, podría estar a la base de la reaparición o exacerbación de síntomas que normalmente se observa en los pacientes fóbicos cuando se les expone nuevamente a los eventos traumáticos (LaBar y Phelps, 2005). De acuerdo a los resultados de la presente investigación y al corpus predominante de información empírica en la actualidad, las asociaciones que se forman entre el contexto y el evento traumático son una de las principales determinantes de las reacciones futuras de los pacientes que todo terapeuta debería considerar.

\section{Referencias}

Allan, L. (1993). Human contingency judgements: Rule based or associative? Psychological Bulletin, 114, 436- 448.

Arcediano, F., Ortega, N., y Matute, H. (1996). A behavioural preparation for the study of human pavlovian conditioning. The Quarterly Journal of Experimental Psychology, 49b, 270-283.

Azorlosa, J., Johnson, C., y McConnell, J. (2006). Acquisition and extinction of conditioned nicotine analgesic tolerance. Learning \& Memory, $34,262-268$.

Barad, M. (2006). Is extinction of fear erasure or inhibition? Why both, of course. Learning \& Memory, 13, 108-109.

Bouton, M. (2004). Context and behavioral processes in extinction. Learning \& Memory, 11, 485-494.

Bouton, M., y Bolles, R. (1979). Role of contextual stimuli in reinstatement of extinguished fear. Journal of Experimental Psychology: Animal Behavior Processes, 5, 368-378.

Bouton, M., y King, D. (1983). Contextual control of the extinction of conditioned fear: Test for the associative value of the context. Journal of Experimental Psychology: Animal Behavior Processes, 9, 248-265.

Cain, C. K., Godsil, B. P., Jami, S., y Barad, M. (2005). The L-Type calcium channel blocker nifedipine impairs extinction, but not reduced contingency effects, in mice. Learning and Memory, 12, 277-284.
Cannistraro, P., y Rauch, S. (2003). Neural circuitry of anxiety: Evidence from structural and functional neuroimaging studies. Psychopharmacol, 37, 8- 25 .

Cheng, P., y Holyoak, K. (1995). Complex adaptive systems as intuitive statisticians: Causality, contingency, and prediction. En Meyer, J., y Roitblat, H. (eds.). Comparative approaches to cognition, 271-302. Cambridge, MA: MIT Press.

Dickinson, A., Shanks, D., y Evenden, J. (1984). Judgement of act-outcome contingency: The role of selective attribution. Quarterly Journal of Experimental Psychology, 36A, 29- 50.

García-Gutiérrez, A., y Rosas, J. (2003). Context change as the mechanism of reinstatement in causal learning. Journal of Experimental Psychology: Animal Behavior Processes, 29, 292-310.

Havermans, R., Mulkens, S., Nederkoorn, C., y Jansen, A. (2007). The efficacy of cue exposure with response prevention in extinguishing drug and alcohol cue reactivity. Behavioral Interventions, 22, 121- 135.

Hendry, J. S. (1982). Summation of undefected excitation following extinction of the CER. Animal Learning Behavior, 10, 476-482.

Kelso, S., y Brown, T. (1986). Differential conditioning of associative synaptic enhancement in hippocampal brain slices. Science, 232, 86- 86.

La Bar, K., y Phelps, E. (2005). Reinstatement of conditioned fear in humans is context dependent and impaired in amnesia. Behavioral Neuroscience, 119, 677-686.

Mao, S. C., Hsiao, Y. H., y Gean, P. W. (2006). Extinction training in conjunction with a partial agonist of the glycine site on the NMDA receptor erases memory trace. Journal of Neuroscience, 26, 8892-8899.

Myers, K. y Davis, M. (2007). Mechanisms of fear extinction. Molecular Psychiatry, 12, 120-150.

Myers, K., Ressler, K., y Davis, M. (2006). Different mechanisms of fear extinction dependent on length of time since fear acquisition. Learning \& Memory, 13, 216-223.

Norrholm, S., Jovanovic, T., Vervliet, B., Myers, K., Davis, M., Rothbaum, B., y Duncan, E. (2006). Conditioned fear extinction and reinstatement in a human fear-potentiated startle paradigm. Learning \& Memory, $13,681-685$.

Pearce, J., y Hall, G. (1980). A model for Pavlovian learning: Variations in the effectiveness of conditioned but not unconditioned stimuli. PSychological Review, 87, 532-552.

Rachman, S. (1989). The return of fear: review and prospect. Clinical Psychology Review, 9, 147-168.

Reberg, D. (1972). Compound test for excitation in early acquisition and after prolonged extinction of conditioned suppression. Learning and Motivation, 3, 246-258.

Rescorla, R., y Heth, C. (1975). Reinstatement of fear to an extinguished conditioned stimulus. Journal of Experimental Psychology: Animal Behavior Processes, 1, 88-96.

Rescorla, R., y Wagner, A. (1972). A theory of Pavlovian conditioning: Variations in the effectiveness of reinforcement and nonreinforcement. En Black, A., y Prokasky, W. (Eds.). Classical Conditioning II: Current Theory and Research. New York: Appleton-Century-Crofts.

Vila, J. (2000). Extinción e Inhibición en juicios de causalidad. Psicológica, 21, 257-273

Vila, J., y Rosas, J. (2001). Reinstatement of acquisition performance by the presentation of the outcome after extinction in causality judgments. Behavioural Processes, 56, 147-154

Wagner, A. (1981). SOP: A model of automatic memory processing in animal behavior. En Spear, N. E., y Miller, R. R. (Eds.). Information processing in animals: Memory mechanisms. Erlbaum: Hillsdale. 